\title{
Memorias de titulación en el marco de la producción en sociología hoy
}

\author{
Juan Pablo Pinilla \\ Francisco Godoy \\ Tania Manríquez \\ Luis Vargas \\ Bernardita Ihnen*
}

\begin{abstract}
Resumen
En el marco de un proceso de reflexividad y autoobservación en las ciencias sociales, el presente artículo busca analizar los rasgos que caracterizan la producción de memorias de titulación en la carrera de sociología de la Universidad de Chile. Con este propósito, aborda el problema de cómo situar los trabajos de habilitación profesional en el contexto de la producción sociológica general, además de identificar tendencias que puedan ser reflejo del desenvolvimiento interno de la disciplina, y de sus cambios institucionales, políticos y sociales. Se sugiere que las tesis constituyen publicaciones claves para el estudio de las comunicaciones científicas, en la medida que involucran aspectos intelectuales del sistema ciencia, junto con dinámicas organizacionales y culturales propias de unidades académicas situadas. Su análisis refleja además que las tendencias en la producción de memorias dice menos relación con un desarrollo multiparadigmático de la sociología, que con criterios de adecuación teórica y pragmatismo metodológico, consistentes con agendas de trabajo progresivas, promovidas según intereses de sus practicantes, docentes o tesistas.
\end{abstract}

Palabras clave: Reflexividad en ciencias sociales - sociología - memorias de titulación - temáticas y contenidos.

\begin{abstract}
In the context of a reflexivity and auto-observation process in social sciences, the present article seeks to analyze the features that characterize the production of undergraduate thesis in sociology at Universidad de Chile. With this purpose, it approaches the problem of how to situate the professional habilitation works in the context of general sociological production, along with identifying tendencies that might be the reflex of the discipline's intern developing and its institutional, politic and social changes. It's suggested that undergraduate thesis constitute key publications for
\end{abstract}

* Juan Pablo Pinilla, Licenciado en Antropología Social de la Universidad de Chile, cursa el magíster en análisis sistémico aplicado a la sociedad en la misma Universidad. E-mail: juan.pablo.pinilla@gmail.com

Francisco Godoy, Licenciado en Antropología Social de la Universidad de Chile. E-mail: f g.31.7h@gmail.com

Tania Manríquez Roa, Licenciada en Antropología Social de la Universidad de Chile. E-mail: taniamanriquez_@hotmail.com

Luis Hernán Vargas Faulbaum, Licenciado en Sociología de la Universidad de Chile. E-mail: ihvargasfaulbaum@gmail.com

Bernardita Ihnen, estudiante de Sociología de la Universidad de Chile. E-mail: berni.ihnen@gmail.com 
the research of scientific communications, insofar as they involve intellectual aspects of the science system, along with the academicals units' own organizational and cultural dynamics. Their analysis also reflects that the tendencies in undergraduate thesis production show a lower relation with a multiparadigmatic development of sociology, than with the theoretical and pragmatic adjustment criteria, consistent with progressive work agendas, situated according to the interests of interns, academics or undergraduate students.

Keywords: Reflexivity in social science - sociology - undergraduate thesis - subjects and contents.

\section{INTRODUCCIÓN}

Numerosos estudios han dado cuenta del carácter reflexivo que caracteriza a la producción en ciencias sociales en la actualidad. Durante el último tiempo, la publicación de investigaciones que buscan objetivar -y con esto, desnaturalizar y volver contingente- los saberes generados al interior de las disciplinas sociales es indicativa de una agenda de investigación que, poco a poco, comienza a tomar curso propio en las comunidades académicas nacionales.

Con todo, el movimiento no ha sido homogéneo ni unitario. Tomando prestadas las conceptualizaciones que Bunge (1993) ha elaborado en sociología de la ciencia, es posible discernir al menos dos tradiciones en el campo de investigaciones nacionales, según la forma en que éstas se aproximan a su objeto de análisis. Por un lado, se encuentran las perspectivas "externalistas", donde el contenido de las actividades científicas se observa condicionado e incluso determinado por los marcos de referencia en que se desenvuelven. Consecuentes con esta línea son estudios sobre el origen, evolución histórica y el contexto sociopolítico en que se enmarcan las ciencias sociales (Arnold, 2009). Casos de externalismo, aunque con un matiz moderado o débil, corresponden a los trabajos de Barrios y Brunner (1988) y Brunner (1988) en torno al marco institucional de la disciplina y sus practicantes; Baño (1984) sobre las tendencias temáticas de la sociología en los ochenta; y Garretón $(1995,2005)$ respecto a los procesos sociopolíticos que contextualizan la evolución de la disciplina. Podría indicarse, además, que mientras los dos primeros trabajos presentan una dimensión "local", donde la comunidad científica concreta es capaz de influir en el trabajo de sus integrantes, los siguientes tres privilegian una visión "global", asociada al énfasis en los condicionamientos de la sociedad en general entre los practicantes de las ciencias (Bunge, 1993: 39).

Del otro lado tenemos una perspectiva "internalista", que describe el campo científico a partir de lógicas definidas desde la misma ciencia, sea a través de la acumulación de conocimiento, los cambios paradigmáticos, la emergencia de programas progresivos de investigación u otras operaciones. A esta línea suscriben los trabajos de Ramos (2005) sobre los agrupamientos paradigmáticos de las investigaciones sociológicas; Ramos et al. (2008) en relación al campo de producción de las ciencias sociales nacionales (sociología, antropología social, ciencia política); Arnold et al. (1990) en relación a las memorias de titulación en antropología social; y Farías (2004) para la sociología de los noventa.

En medio de la agenda de trabajo que se ha ido conformando, el artículo busca analizar los rasgos que caracterizan la producción de memorias de titulación en la carrera de sociología 
de la Universidad de Chile ${ }^{1}$. Con este objeto, hace suyo el problema de cómo situar los trabajos de habilitación profesional en el contexto de la producción sociológica general, intentando identificar tendencias temáticas, metodológicas y teóricas que potencialmente puedan reflejar el desenvolvimiento interno de la disciplina. Del mismo modo, interpretar estos productos desde el punto de vista de las condiciones organizacionales y culturales del campo académico concreto en que son elaborados.

El texto se organiza en seis apartados. El primero de ellos expone el aparataje conceptual que es utilizado en la interpretación y discusión de los datos, en tanto el segundo sintetiza los antecedentes del proceso de institucionalización y profesionalización de la sociología en el país (con especial atención a su quehacer académico durante las últimas dos décadas). Posteriormente se expone el aparataje conceptual que es utilizado en la interpretación de los datos, para luego continuar con una presentación de los principales resultados de la investigación. Los datos provenientes de 220 memorias de título presentadas en la carrera de sociología de la Universidad de Chile entre 1988 y 2007, son examinados en su constitución metodológica, teórica y temática. De los resultados se desprenden procesos de emergencia y decadencia de ciertas áreas de estudio, así como condiciones institucionales que son relevantes para el trabajo de los practicantes de la disciplina. Finalmente, se discute acerca del valor de estos productos (las memorias de titulación) en el seno de la producción sociológica, y su cualidad indicativa de procesos externos al ámbito del sistema ciencia.

\section{MODELOS PARA ESTUDIAR LA PRODUCCIÓN EN SOCIOLOGÍA}

Múltiples son los recursos teóricos disponibles para adentrarse en el campo de la reflexividad de las ciencias sociales, así como para la interpretación de su elaboración científica y su reproducción institucional. Allende la extendida oposición entre enfoques internalistas y externalistas (Bunge, 1993; Bourdieu, 2000b), para comprender la producción de conocimiento científico existen al menos cuatro grandes modelos en disputa, cuya exposición y elaboración integrativa han sido extensamente tratadas por Claudio Ramos (2008). Para el autor, los modelos conceptuales en juego corresponderían a (1) los campos científicos de Bourdieu; (2) la tradición sociocultural y el Programa Fuerte de Shapin, Knorr-Cetina, Pickering, Lynch y Traweek; (3) la teoría del actor red representada por Latour, Callon y Law; y (4) la teoría de sistemas de inspiración luhmanniana. Sin ser parte de nuestro cometido una exposición acabada de cada uno de ellos, en lo que sigue, diseñaremos un esquema conceptual sencillo que, incorporando distinciones de diversas tradiciones, aspira a constituir un corpus adecuado para el análisis e interpretación de los datos levantados.

Las claras fronteras que Weber (1967) trazó entre la actividad científica y la actividad política del sociólogo profesional presentan hoy en día complicaciones sustantivas. Como

1 El texto forma parte de la investigación Memorias de titulación en antropología social y sociología de la Universidad de Chile, 1988-2007, financiada por el Centro Multidisciplinario de Investigación Social y Cultural PULSO, de la Facultad de Ciencias Sociales de la Universidad de Chile. Los resultados obtenidos en el estudio se presentan en extenso en Pinilla et al. (inédito). 
ha constatado Bourdieu (2000b), las ciencias sociales deben convivir con la imposibilidad de reclamar con absoluta legitimidad la hegemonía sobre las representaciones científicas del mundo social, teniendo que hacer frente no sólo a otros científicos, sino también a los profesionales de las representaciones simbólicas (escritores, políticos, periodistas) que intentan imponer su visión del objeto. Más radicales son las perspectivas sistémico constructivistas, según las cuales el obstáculo ineludible que enfrentan estas disciplinas, y la ciencia en general, es la evidencia de que sus productos son resultado de "construcciones de un observador que se encuentra siempre imposibilitado de contactarse directamente con su entorno" (Arnold, 2004: 9).

Con el propósito de aprehender teóricamente el movedizo campo en que se mueve la producción sociológica, es preciso dar cuenta primero del dominio fenoménico suscrito bajo la noción de disciplina científica.

Siguiendo de cerca a Wallerstein (1999), observamos que el término "disciplina" estaría aludiendo a tres ámbitos distintos: (a) el dominio intelectual, como la dimensión cognitiva del conocimiento; (b) el dominio organizacional, en tanto estructuras corporativas; y (c) el dominio cultural, respecto a las comunidades de estudiosos que comparten ciertas premisas elementales.

El primer campo aludiría a una "circunscripción intelectual de conocimiento, un conjunto de tópicos y métodos diseñados para discutir un ámbito delimitado de fenómenos del mundo real" (Wallerstein, 2004: 22). Sin dudas es el ámbito de mayor abstracción teórica, donde la sociología es vista como un "artefacto heurístico" que reclama para sí un ámbito de estudio, con su región particular, sus métodos propios y sus fronteras (Wallerstein, 1999: 11). Caben aquí proposiciones acerca del sistema científico en general, y el tratamiento de las ciencias sociales como una súper disciplina subdividida a su vez en una serie de disciplinas específicas.

Desde un punto de vista institucional, las disciplinas son también contenedores organizacionales, que a través del tiempo han sabido estructurarse al alero de centros universitarios, en facultades (súper disciplinas) y en departamentos (disciplinas). Estas unidades constituyen para Wallerstein (2004) contenedores con recursos y poder, por lo que han buscado definir cada vez más claramente el contenido intelectual de la 'disciplina' que contienen, sea a través de pautas metodológicas que definen lo que está dentro y fuera del campo científico, como por medio de la definición de mallas curriculares de las asignaturas. En un sentido amplio, estas y otras instancias operan como mecanismos de reproducción de una unidad disciplinar.

Las disciplinas se manifiestan, por último, como comunidades culturales, originadas en tanto las tendencias intelectuales tienden a favorecer ciertos tipos de investigación, ciertos métodos y lecturas compartidas. Se expresan, en palabras de Barrios y Brunner (1988: 11), como "profesionales intelectuales", que se mueven dentro de marcos gnoseológicos o "paradigmas", compuestos tanto por los ideales explicativos y los modelos conceptuales -la constelación de creencias- comunes a los miembros de una comunidad, así como por personas con formaciones similares y que intercambian experiencias en su especialidad. Es 
parte de este ámbito, además, el saber-hacer orientado al bien común propio a las profesiones, y su engarce con las demandas del mundo del trabajo y requerimientos de empleadores y usuarios de los servicios profesionales (Farías, 2009: 61).

Una aproximación consistente a la dimensión intelectual de la academia es la que provee el programa sistémico representado por Luhmann. Ramos ha visto, de modo a nuestro juicio veraz, que el modelo sistémico presenta buenos rendimientos para aprehender "la dinámica cognitiva de la ciencia, su proceso de autonomización y diferenciación" (Ramos, 2008: 24). La arquitectura teórica luhmanniana parte de la concepción de una sociedad compleja, diferenciada en base a la especialización de funciones parciales, donde la ciencia no puede abogar para sí una visión privilegiada sobre la totalidad social. No pudiendo suponerse una naturaleza social unitaria y un único medio para su observación, las descripciones unilaterales, propias de un mundo monocontextual, quedan obsoletas (Arnold, 2006: 323).

En el marco de teoría sistémica la ciencia puede ser entendida como una empresa de la sociedad que produce conocimiento; o, más precisamente, como un sistema funcional de la sociedad. Como señala Luhmann (1996: 11), "describimos la ciencia como un sistema funcional de la sociedad moderna que ha podido diferenciarse bajo condiciones históricas del marco social y convertirse en una unidad operativa propia, es decir, en condiciones para diferenciar lo verdadero de lo que no lo es". Es así que, en base a un código binario que distingue entre lo verdadero y lo no verdadero, el subsistema parcial de la ciencia logra clausura en sus operaciones. Opera recursivamente por cuanto es capaz de aplicar sus operaciones al producto de operaciones anteriores, de modo tal que todas las comunicaciones, de aquí en adelante, podrán ser procesadas en uno de los polos de la distinción (verdad/no verdad).

La cerrazón del sistema ciencia tiene por consecuencia que ningún criterio externo a ella tenga valor rector sobre las decisiones que se toman sobre sus operaciones comunicativas. Las selecciones de conocimientos se producen a través de la acción de "programas" científicos, que varían en el tiempo y que tienen por finalidad la aplicación del código a comunicaciones con pretensiones científicas (Ramos, 2008: 21). Una de estas formas de autovalidación del saber corresponde a las publicaciones de textos, condición de la autopoiesis del sistema. En efecto, son el texto y su medio de publicación mecanismos fundamentales para concebir la producción científica en Luhmann (1996), ya que sólo así es pensable el establecimiento de una red comunicativa autopoiética en torno a los productos de la investigación en ciencias.

En nuestro caso, las memorias de titulación constituyen materiales particulares en la producción científica con fines comunicativos. Según Martínez y Ríos, un trabajo de grado o tesis puede entenderse como: "producto de una investigación teórica o práctica, que puede estar orientado a la generación de teoría o a la aplicación de la misma, dentro de un campo científico particular, y que tiene como propósito final el ampliar el conocimiento en ese campo y el acreditar al estudiante o participante de un programa de estudio con un título o grado académico" (Martínez y Ríos, 2006: 10).

La forma en que se enmarca el proceso de elaboración de las memorias de título hace que estas establezcan, de un lado, un vínculo especial con tendencias paradigmáticas o 
programáticas del sistema ciencia. Así sucede con ciertas modas teóricas, marcos epistemológicos emergentes, saltos paradigmáticos o nuevos programas de investigación. Sin embargo, el carácter iniciático y novel que caracteriza el proceso investigativo de las tesis, sean éstas de tipo abstracto o aplicado, ponen atajos a la plena autonomía del practicante. La presencia de tutores guías y la defensa del trabajo frente a una comisión de docentes de la unidad imprimen en el producto importantes rasgos de la dimensión organizacional y cultural de la unidad académica situada. Es por esto que, junto con entender el valor comunicativo de las memorias en tanto publicaciones científicamente validadas en sus comunidades de origen (en este caso, la carrera de sociología de la Universidad de Chile), sea preciso además contar con herramientas teóricas de menor altura, con tal de observar dinámicas propias del campo científico en una institución.

Es preciso hacer hincapié en el peso de esta dimensión, de manera tal de consignar procesos de las unidades académicas concretas. Como asevera Wallerstein: "La institucionalización de una disciplina es una vía de preservar y reproducir prácticas. Representa la creación de una auténtica red humana con fronteras, una red que asume la forma de estructuras corporativas que tienen requisitos de ingreso y códigos que proporcionan caminos reconocidos para la movilidad profesional ascendente" (Wallerstein, 1999: 13).

Probablemente sea el modelo elaborado por Pierre Bourdieu una de las herramientas más potentes para observar la dimensión organizacional involucrada en la producción de saberes científicos ${ }^{2}$. Bourdieu parte su análisis por las condiciones sociales (y sus mecanismos genéricos) que rigen un campo de aceptación de nuevos productores, cuyas elaboraciones puedan ser observadas como objetos "relativamente independientes de sus condiciones sociales de producción" (Bourdieu, 2000a: 75). Tales productos son lo que el autor denomina "verdades científicas". La ciencia es aquí un campo social como cualquier otro, con relaciones de fuerza y monopolios en sus dinámicas, con luchas y estrategias de agentes interesados ${ }^{3}$.

El campo científico es por sobre todo un campo de producción simbólica (Ramos, 2008: 25); un sistema de relaciones objetivas entre posiciones adquiridas en luchas anteriores (Bourdieu, 2000a: 76). A él concurren agentes que aspiran al monopolio de la autoridad científica, capacidad que se ofrece tanto a un saber técnico como a un poder social. El monopolio de la competencia científica puede ser visto como una estrategia para aumentar

2 No desarrollaremos aquí los siguientes dos modelos identificados por Ramos (2008), correspondientes al Programa Fuerte y la perspectiva sociocultural, y la teoría del actor red. La concentración del artículo en el producto de las Memorias, y la escasez de evidencias referidas al proceso de su construcción, nos hacen privilegiar modelos generales como el de Luhmann, junto a añadidos del campo organizacional de las unidades académicas otorgado por Bourdieu.

3 Sabido es que Bourdieu otorga a las ciencias sociales una posición ambigua en el campo científico. Para el autor, tales disciplinas no dispondrían sino de una falsa autonomía, por contraposición a las ciencias naturales donde el objeto de estudio no va en directo interés de los grupos dominantes. De ello deriva el hecho de que "las posiciones en la lucha interna [del campo científico] no puedan esperar jamás el grado de independencia respecto a las posiciones en la lucha externa que se observa en el campo de las ciencias de la naturaleza" (Bourdieu, 2000a:101ss). 
el capital de los agentes, resultado de actos de conocimiento y reconocimiento del medio (Ramos, 2008: 26).

En más de un aspecto, podemos afirmar que la posesión de un título profesional constituye un factor de incremento del capital social en un practicante de sociología, lo que potencialmente puede reflejarse en un incremento de sus remuneraciones (Cf. Bourdieu, 2000a: 88). A esto refiere el hecho de que el título, en tanto que certificación de un capital escolar, pueda ser reconvertido en capital universitario y científico, marcando el rumbo de una trayectoria probable de la carrera científica.

Pero el valor de la producción de memorias de título no se acaba en estas circunstancias del campo científico. La movilización de los practicantes (tesistas) a través de "estrategias de sucesión" y "estrategias de subversión", permite a los noveles investigadores asegurarles bien la realización del "ideal oficial de la excelencia científica al precio de innovaciones circunscritas a los límites autorizados", bien la costosa colocación de una "redefinición completa de los principios de legitimación de la dominación" en el campo en cuestión (Bourdieu, 2000a: 93). Con todo, existe una tercera vía que Bourdieu no consigna con suficiente atención, y que representa una estrategia adaptativa-digresiva donde los practicantes abren gradualmente líneas de trabajo alternativas que, sin afectar directamente el orden estatuido del campo, van incorporando nuevos agentes al juego y paulatinamente alterando las distribuciones de capitales al interior del mismo.

\section{EL PANORAMA DE LA SOCIOLOGÍA CHILENA ACTUAL}

Parte importante de los trabajos que versan sobre el desarrollo de la sociología en América Latina y en Chile (Baño, 1984; Barrios y Brunner, 1988; Brunner, 1988; De Sierra et al., 2005; Garretón, 2005) han puesto énfasis en la relación existente entre el proceso de institucionalización académica de la disciplina y el entorno sociopolítico de la región; desde una perspectiva externalista que se empeña en mostrar cómo es que el medio logra modelar el trabajo de los practicantes de la ciencia, ejerciendo una influencia significativa en la selección y construcción de su objeto de estudio. Dentro de los recuentos históricos, destacan los aportes de Manuel Antonio Garretón (2005) y su propuesta de tres períodos para trazar la evolución de la academia sociológica en Chile. Para Garretón, en este curso se distingue (1) una fase fundacional de la disciplina, donde la sociología se visualiza como un proyecto académico-político; (2) una fase de ruptura, donde las iniciativas y principios del momento anterior son puestos en tela de juicio, marcada por la irrupción de las Fuerzas Armadas y los correspondientes golpes de Estado en casi todo el Cono Sur; y (3) una fase de transición a la democracia, coincidente con el renacimiento institucional de la sociología y con la consolidación de enfoques de investigación alternativos que aparecen a mediados de la década del ochenta.

En lo que a la investigación compete, nos centraremos exclusivamente en esta última etapa, atendiendo a los rasgos que caracterizarían la producción sociológica de los últimos veinte años, y los marcos institucionales, políticos y sociales en que se desenvuelve. Tal vez la transformación más importante diga relación con el tránsito de una sociología que durante 
los 60 y comienzos de los 70 comenzaba a polarizar sus discursos críticos, envolviéndose estrechamente en las querellas político-ideológicas del momento (Brunner, 1989: 5), hacia una disciplina que, en términos globales, estructura su campo de competencias "en torno a dinámicas de mercado, tanto en el ámbito de la docencia como en el de la investigación" (Courard y Frohmann, 1999: 55).

Es sintomática de este giro la inicial carencia de esfuerzos colectivos en el seno de las academias sociológicas chilenas, lo que en un primer momento se ve subsanado por esfuerzos individuales concentrados en temas particulares, que darán pauta a una creciente especialización temática que se extenderá en la década de los 90 . Entre las investigaciones empíricas disponibles para esta fase, Garretón y Mella (1995) han observado ciertas dimensiones que serían predominantes en la sociología de aquellos años. Entre sus rasgos más sobresalientes destaca un marco general de tipo metodológico asociado a técnicas tanto cuantitativas como cualitativas; y un marco teórico o temático que apunta al estudio del Chile postautoritario, mediante una profundización de los procesos democráticos y una redefinición de la modernidad. Se busca dar así con nuevos modelos heurísticos para comprender un estilo de desarrollo distinto -el neoliberal-, haciendo énfasis en el fenómeno de inserción internacional de Chile en el proceso de globalización. Atrás quedan los términos de "subdesarrollo" y "procesos de modernización" como conceptos límites del campo investigativo (Brunner, 1989: 5), para dar paso a ámbitos de especialización vinculados a una sociología de la vida cotidiana, de la familia, de las comunicaciones de masas, rural y vinculada a estudios étnicos (Garretón y Mella, 1995). En palabras de Baño, surge una "preocupación por lo cotidiano, esto es, por los hechos rutinarios que no constituyen acontecimientos" (Baño, 1984: 11).

En el ámbito institucional se observa también una recomposición y reestructuración del campo de las ciencias sociales en general, y de la sociología en particular. Por ejemplo, se aprecia para este momento una expansión importante de las matrículas universitarias. Prueba de ello es que en 1990 existían tres carreras de sociología a lo largo del país, mientras que para 1995 dicha suma llegaba a ocho programas de pregrado (Universidad de Chile, Pontificia Universidad Católica, Universidad Academia de Humanismo Cristiano, Universidad de la República, Universidad Arturo Prat, Universidad de La Frontera, Universidad ARCIS y Universidad de Concepción), los cuales reunían a 1.120 alumnos matriculados.

Por su parte, Ramos sostiene que en dicho período se habrían visto involucrados diversos cambios que afectaron a los productores de conocimiento social, vinculándose tanto a las redes y canales de comunicación así como al origen de los fondos para investigación. Entre estas tendencias, se menciona que "i) se restablecen las comunicaciones intracampo, reconectándose investigadores antes separados en ONGs, el Estado y las universidades; ii) cambian las pautas de financiamiento: disminuyen sustancialmente los fondos internacionales y crece el financiamiento de la investigación social desde el Estado; y iii) se intensifica la búsqueda por generar conocimientos usables y gestionables para fines prácticos, tanto en instancias públicas como privadas". (Ramos, 2005: 92). A ello se suma el fenómeno de los think tanks y centros académicos privados, que sirven de medio para desarrollar la actividad ideológica y técnica de expositores de la sociología chilena (Brunner, 1985). 
Así, en términos institucionales, la sociología vuelve a estar presente en las universidades sin interrupciones, al igual que en el aparato estatal, por lo que el financiamiento externo para los centros académicos independientes disminuye significativamente en este período. Pese al papel que juega ahora el mercado, parte importante de la investigación básica y aplicada es sostenida económicamente por medio de fondos concursables financiados por el Estado a través de los Conicyt (Fernández, 2007).

Otra característica importante será la constatación de una ausencia de paradigmas únicos u omnicomprensivos para el abordaje de fenómenos sociales, siendo relevante el grado de diversificación que presenta la disciplina. Se constata un panorama "en este sector de las ciencias sociales, caracterizado por la existencia de múltiples tendencias centrífugas. Ya no hay un centro temático configurado en torno a unos pocos tópicos y debates, bajo la hegemonía de una escuela o teoría predominante" (Brunner, 1989: 10). Si bien esta diversificación no es problemática, en ciertos contextos puede traducirse en la incomunicación de las distintas partes, lo que atenta contra el desarrollo del campo sociológico (Cf. Bourdieu, 2000c: 120ss).

Respecto a las tendencias observadas en la investigación sociológica reciente, Ramos ha sostenido que es posible comprender a la sociología chilena como un campo multiparadigmático, en el cual "existen claras y marcadas diferencias epistemológicas y ontológicas, con una acumulación que se produce dentro de cada paradigma, y con claros síntomas de inconmensurabilidad entre paradigmas, cada uno con sus propios procedimientos aceptables, su propia noción de lo que es una prueba empírica y su propia concepción de lo que constituye conocimiento válido" (Ramos, 2005: 87). Luego de un proceso de registro y clasificación en variables de 105 obras sociológicas producidas entre 2000 y 2004, y un subsiguiente análisis por conglomerados, el autor reconoce cuatro agrupamientos paradigmáticos, dos de los cuales pueden ser considerados como variantes del postpositivismo, y las restantes como variantes del paradigma interpretativo (Ibíd: 96-97)4. El primero de ellos es denominado "postpositivista fuerte", y usa con mayor sofisticación los procedimientos de análisis cuantitativo, por contraposición al "postpositivista débil", que presenta menor complejidad en sus análisis y menor consistencia interna. Por el otro lado encontramos al "interpretativo fuerte", el cual se focaliza en procesos de interpretación y es más coherente en su tratamiento de la realidad como realidad significativa respecto a lo que realiza el conjunto "interpretativo híbrido", que combina procedimientos cuantitativos e interpretativos y atiende tanto a aspectos de sentido como a regularidades objetivas.

4 Por razones de espacio no podemos abundar respecto a los elementos concluyentes en la construcción de estos agrupamientos paradigmáticos, no obstante lo cual mencionaremos las seis variables utilizadas por el autor para realizar el registro y clasificación de su corpus: 1) tipo de supuestos sobre el carácter de la realidad; 2) forma en que son abordados los procesos de tipo causal; 3) presencia y rol de valores extracientíficos que orientan la investigación; 4) tipo de conceptualización de la realidad; 5) empleo o no de documentos como fuente para la indagación empírica; 6) empleo o no de encuestas como instrumento para la generación de información (Ibíd: 96). 
En este sentido, las conclusiones del mentado estudio afirman que "la investigación sociológico-empírica de principios del siglo XXI en Chile sigue estando fuertemente orientada y marcada por el positivismo, sea por las potencialidades instrumentales que posee esta forma de producción de conocimientos, o por la concordancia con las creencias sociales imperantes, o por la lentitud en las instituciones formadoras para acoger nuevas formas de investigación" (Ibíd: 116). Hasta cierto punto es factible sostener que estos aspectos constituyen elementos de la disciplina sociológica en tanto comunidad cultural (Wallerstein, 1999), constituidos por supuestos tácitos o inconscientes que tienden a favorecer ciertos tipos de investigación, ciertos tipos de métodos, y ciertas lecturas compartidas.

Por otro lado, las herramientas metodológicas detectadas por Ramos muestran "la prevalencia de la encuesta, asociada a la continuidad positivista, junto con una mayor flexibilidad y eclecticismo en el uso de técnicas, el cual se manifiesta en un generalizado uso simultáneo de varias técnicas de recolección de información" (Ramos, 2005: 118). Cabe acotar que la utilización de encuestas, junto con la referencia a estadísticas levantadas por medio de ellas, está presente en el 70\% de las obras analizadas por el autor, lo que la define como la "herramienta sociológica reinante en la actualidad".

El trabajo de Fernando Farías (2004) sobre la producción sociológica durante la década del 90 aporta datos relevantes respecto a la distribución de la disciplina en áreas temáticas (subdisciplinas), mostrándose un aporte mayoritario en sociología de la cultura $(21,8 \%)$, sociología del trabajo (14,5\%) y la sociología política (14,5\%). En cambio, las áreas con menor cantidad de trabajos publicados son la sociología matemática, experimental y problemas internacionales, todas éstas con un aporte de 1,4\% a la productividad total de publicaciones.

Las instituciones más productivas del país son la Facultad Latinoamericana de Ciencias Sociales (FLACSO) con un 26,1\%, seguido por la Universidad de Chile (UCH) con un porcentaje de un 26,0\% y la Pontificia Universidad Católica de Chile (PUCCH) (12,4\%). A partir del registro de las palabras más utilizadas en los trabajos publicados, es posible observar que en su mayor parte dichas obras hacen alusión a la región latinoamericana en primera instancia, y a Chile en segundo lugar; las principales áreas temáticas referidas son política y cultura, y en cuanto a temas específicos destacan los de "desarrollo" y "democracia" (Ibíd).

A modo de resumen, es posible colegir tres ideas que caracterizarían el desarrollo disciplinar de la sociología chilena en los últimos veinte años:

a. En el ámbito intelectual, la sociología ha pasado por la instalación de nuevos modelos en el hacer científico. En general, estos se han traducido en el surgimiento de agendas de investigación cuyos focos se asocian a una diversificación y especialización temática, con un decaimiento del paradigma crítico, el fortalecimiento del postpositivismo y un cierto marco interpretativo.

b. En el ámbito organizacional, con la reapertura de carreras y conformación de nuevas unidades académicas se produce un fortalecimiento de la comunidad sociológica. Se 
evidencia además el establecimiento de relaciones con el mercado, aunque conservando vínculos entre universidades, con el Estado y con centros de investigación privados.

c. Desde el punto de vista cultural hay una cierta pérdida de unidad disciplinar o "desdibujamiento" (Wallerstein, 1999), donde las fronteras disciplinares y sus líneas de investigación tradicionales dejan de operar como sentidos claros y definidos de antemano en la práctica sociológica. Esto abre espacio a innovaciones y a cambios en la identidad de la disciplina.

\section{MÉTODOS E INFORMACIÓN}

Para el análisis de la producción sociológica la investigación se definió como universo de estudio las memorias de titulación producidas en la carrera de sociología en la Universidad de Chile. La elección de la muestra se basó en criterios de relevancia y masividad de los productos, así como su accesibilidad.

Por memoria de título se entendió "un trabajo original que acredita conocimientos teóricos y metodológicos en la disciplina", siendo la "actividad curricular obligatoria para la obtención del Título" en la Universidad de Chile (Artículo 56², Decreto Exento № 022558).

El estudio pretendió identificar los rasgos característicos en la producción de las memorias, en el intervalo temporal que va de 1988 al 2007. La disponibilidad de antecedentes para el período anterior, así como su coincidencia con el proceso de redemocratización en el país, justifican el corte temporal utilizado. La información relativa a la elaboración de tesis fue levantada mediante fichas de registro por cada una de las unidades de estudio. Se aplicó un cuestionario a 220 documentos, con cinco ítems generales: información de la memoria, identificación temática, identificación bibliográfica, identificación metodológica y resultados.

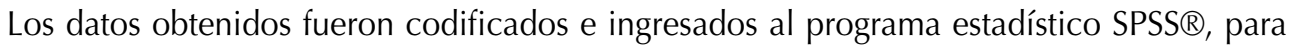
su procesamiento y análisis.

El plan de análisis de los datos incluyó una dimensión abocada a la caracterización de la muestra; otra a sus opciones metodológicas; una tercera a las tendencias temáticas; y finalmente un subapartado sobre las inclinaciones teóricas.

\section{ANÁLISIS Y RESULTADOS}

\subsection{Caracterización general}

La producción de memorias de titulación en la carrera presenta una distribución desigual a lo largo del período analizado (gráfico 1). El promedio de publicaciones fue de 11 documentos anuales, con una significativa dispersión (desviación típica de 7,9). Se aprecia además una tendencia al alza en la producción a partir de 2004, aunque tal aumento no es sostenido año a año, ni se condice con una ampliación importante en el número de matriculados de la carrera. Así, mientras que en el período que va desde 1988 a 2000 la media de memorias es 7, entre 2001 y 2007 ésta asciende a 19 trabajos anuales promedio. 


\section{GRÁFICO 1}

Memorias de titulación según año de publicación

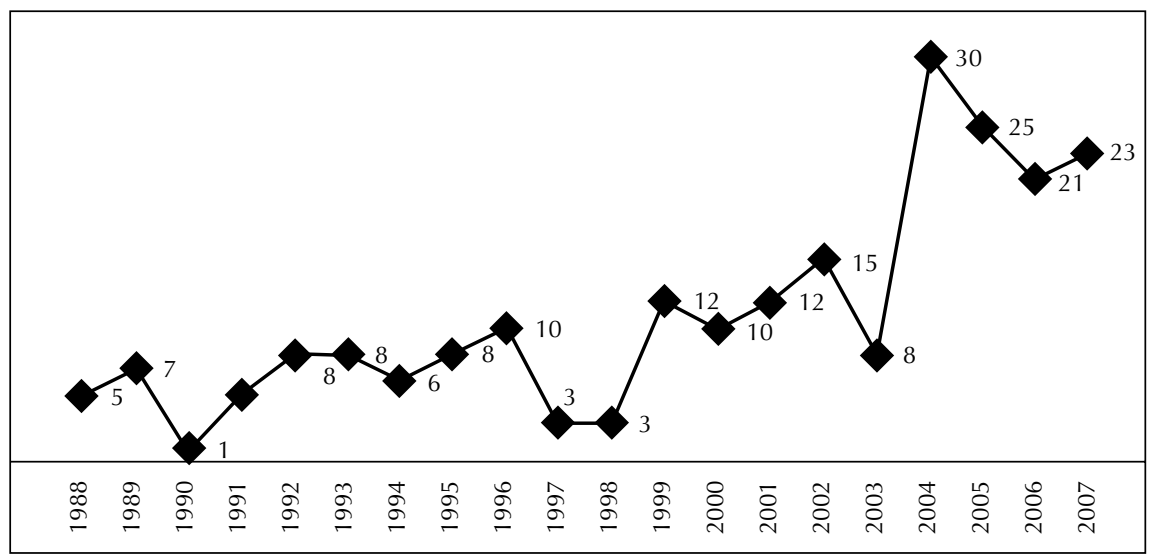

Fuente: elaboración propia.

Desde el punto de vista del sexo de los autores, se observa el ingreso importante de mujeres en la elaboración de tesis; pasando de representar el 35\% de los documentos entre 1988 y 2000, al 50\% de los trabajos publicados desde 2001 a 2007.

\subsection{Metodología}

Es sabido que la utilización de una determinada metodología resulta fundamental, y que es la principal decisión práctica de la cual depende el cumplimiento de los objetivos del trabajo y la respuesta a la pregunta que guía el proceso investigativo. Su relevancia es tal, que podemos afirmar junto con Ramos (2005: 86), que "la índole misma de lo que se observa está condicionada por el aparataje o procedimiento metodológico". Para la revisión de las distintas elecciones de corte metodológico, se utilizó un conjunto de indicadores que abarcaban: tipo de estudio, estrategia de investigación e instrumento de producción de información.

Entre los resultados obtenidos destaca el peso significativo que han adquirido los estudios de carácter exploratorio y descriptivo, los que incluso han ganado espacio ante los trabajos de carácter explicativo y correlacional (tabla 1). Resulta relevante, en este sentido, asociar dicho fenómeno a prácticas de búsqueda y apertura de nuevas áreas de investigación, muy en la línea de la diversificación temática mencionada antes, y que en última instancia se apartan de la pura reproducción disciplinar. No obstante aquello, no debe obviarse el hecho de que esta apertura hacia nuevos espacios implica también -aunque no necesariamente- el dejar de lado caminos ya trazados al interior de la tradición sociológica, lo que en ciertos contextos se expresa en una relativa pérdida de referentes pasados y la apertura hacia variaciones en la identidad disciplinar. 
En la Universidad de Chile, los estudios sociológicos exploratorios abordan preferentemente temáticas urbanas, rurales y medioambientales, en tanto los descriptivos se concentran en campos como la salud y el género, y de carácter rural y urbano. Documentos con aspiraciones explicativas tienden a concentrarse en tópicos asociados a la estratificación y la política, donde existe un corpus de investigaciones importantes a lo largo de la producción sociológica de las últimas cuatro décadas.

Por otra parte, debe resaltarse el hecho de que las estrategias principal y exclusivamente cualitativas son por lejos las más utilizadas en las investigaciones $(52 \%)$, con un notorio aumento en el segundo período (gráfico 2). Tales preferencias van en detrimento de estrategias

TABLA 1

Memorias de titulación según atributos metodológicos, por período (en porcentaje)

\begin{tabular}{|c|c|c|c|c|}
\hline \multirow{2}{*}{\multicolumn{2}{|c|}{ ATRIBUTOS METODOLÓGICOS }} & \multicolumn{3}{|c|}{ PERÍODOS } \\
\hline & & \multirow{2}{*}{$\frac{1988-2000}{16,3}$} & \multirow{2}{*}{$\frac{2001-2007}{16,4}$} & \multirow{2}{*}{$\begin{array}{r}\text { TOTAL } \\
16,4\end{array}$} \\
\hline Tipo de estudio & Teórico, ensayístico & & & \\
\hline & Exploratorio & 48,8 & 54,5 & 52,3 \\
\hline & Descriptivo & 55,8 & 66,4 & 62,3 \\
\hline & Correlacional & 19,8 & 10,4 & 14,1 \\
\hline & Explicativo & 9,3 & 4,5 & 6,4 \\
\hline & Estudio de caso & 9,3 & 13,4 & 11,8 \\
\hline & Investigación evaluativa & 3,5 & 3,0 & 3,2 \\
\hline \multirow[t]{7}{*}{ Tipo de estrategia de inv. } & Teórica, ensayística & 15,1 & 16,4 & 15,9 \\
\hline & Exclusivamente cuantitativa & 17,4 & 14,2 & 15,5 \\
\hline & Exclusivamente cualitativa & 30,2 & 49,3 & 41,8 \\
\hline & Principalmente cuantitativa & 14,0 & 1,5 & 6,4 \\
\hline & Principalmente cualitativa & 8,1 & 12,7 & 10,9 \\
\hline & Mixta & 12,8 & 9,0 & 10,5 \\
\hline & Investigación acción & 2,3 & 1,5 & 1,8 \\
\hline
\end{tabular}

* Las categorías no son mutuamente excluyentes, de modo que no suman $100 \%$.

Fuente: elaboración propia. 


\section{GRÁFICO 2}

Memorias de titulación según estrategia metodológica, por período

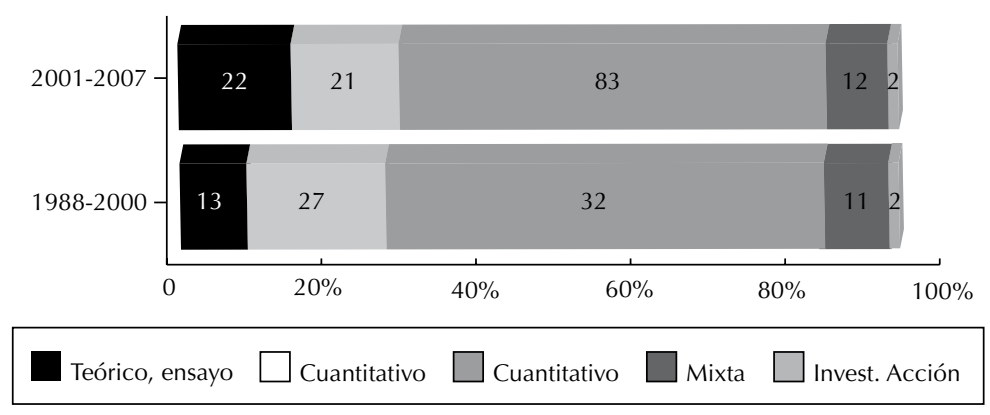

Fuente: elaboración propia.

cuantitativas, las que, atendiendo a la tradición disciplinar, podrían considerarse como opciones metodológicas arraigadas en el medio nacional (Cf. Ramos, 2005).

Lo anterior tiene un correlato con los instrumentos de producción de información privilegiados, donde las entrevistas individuales son por lejos las más utilizadas $(54,1 \%)$, presentando un notorio aumento en el segundo período, al igual que el análisis de contenido. Por otra parte, las encuestas permanecieron rezagadas al tiempo que el uso de datos secundarios cuantitativos aumentó ligeramente. En este punto es preciso plantear que las decisiones metodológicas son adoptadas muy probablemente de modo pragmático, orientadas a dar respuesta del modo más accesible al problema que motiva la investigación; accesibilidad que se define en términos de disponibilidad de recursos (monetarios y logísticos) y no en función de los rasgos característicos de las competencias disciplinares. Es así que la lectura de las memorias reflejaría que un "pragmatismo metodológico" constituye un sentido práctico en el quehacer de los tesistas de la carrera.

Desde otro punto de vista, Farías (2009) ha planteado argumentos congruentes con esta idea respecto al pluralismo metodológico postulado por Paul Feyerabend. El autor señala que las decisiones metodológicas no son adoptadas a priori en la práctica científica, como si atendieran a algún paradigma sólidamente definido, sino que se escogen en función de la situación o problemática planteada. De este modo, se destaca que al reflexionar sobre las estrategias epistemológicas y metodológicas desde la práctica misma se disuelven los dogmatismos ( $I$ bíd). Justamente, esto da pie para sugerir que, al menos a nivel de pregrado, las construcciones metodológicas no son siempre consistentes con modelos paradigmáticos plenamente constituidos e internamente coherentes.

\subsection{Temas}

La identificación temática de las memorias se realizó mediante la clasificación de cada trabajo en una o más de entre 41 categorías cerradas. Cada documento podía así ser inscrito 
en un máximo de 3 categorías, para obtener un indicador sensible a ámbitos de estudio complejos que abarcan más de un tema en particular. Para la posterior interpretación de los resultados, los indicadores temáticos fueron recodificados en 18 temas generales o macrotemas.

Los hallazgos obtenidos muestran que las elecciones temáticas resultan de importancia crítica respecto al futuro desarrollo profesional del tesista, en tanto le permiten insertarse en las diversas agendas temáticas y profesionales que tienen lugar tanto fuera como dentro de la unidad académica de estudio. En este sentido, debe considerarse a la elección temática el producto tanto de las motivaciones individuales de los practicantes, como de los condicionamientos ejercidos por la institución formadora (en este caso, el Departamento de Sociología de la Universidad de Chile, con su particular composición en términos de planta docente y de malla curricular).

En relación al primer ámbito, Bourdieu señala que este tipo de elecciones se realizan "por referencia a una anticipación-sea consciente o inconsciente- de las posibilidades medias de beneficio (que se especifican también en función del capital detentado)" (Bourdieu, 2000a: 78). Tal cálculo permitiría comprender el por qué inscribirse dentro de una línea temática y establecida, o bien buscar nuevos horizontes. En efecto, "la tendencia de los investigadores a concentrarse sobre los problemas considerados como los más importantes (por ejemplo, porque han sido constituidos como tales por productores dotados de un alto grado de legitimidad) se explica por el hecho de que un aporte o un descubrimiento que concierne a estas cuestiones está encaminado a aportar un beneficio simbólico más importante. La competencia intensa que se encuentra así desencadenada tiene todas las posibilidades de determinar una baja de las tasas medias de beneficio y/o simbólico y, por este medio, la dirección de una fracción de los investigadores hacia otros objetos menos prestigiosos pero alrededor de los cuales la competencia es menos fuerte $y$, por ello, adecuados para ofrecer beneficios, al menos, igualmente importantes" (Ibíd).

Por otra parte, y atendiendo a lo señalado por Wallerstein $(1999,2004)$ en referencia a la disciplina en tanto organización (como instancia de reproducción de prácticas), vale la pena revisar cuáles son los principales lineamientos del Departamento de Sociología de la Universidad de Chile. A este nivel, se evidencia que la unidad académica ha vivido un proceso de estructuración en los últimos años, teniendo en un inicio políticas institucionales más bien desarticuladas e inconexas, que luego comienzan a ser revertidas gracias a distintos esfuerzos por alinear la investigación del Departamento bajo una coordinación interna, cuyos hitos más significativos son la Comisión Académica del Departamento de Sociología (2004) y la Memoria Anual de Investigación (2009).

La Comisión del 2004 estableció 4 áreas temáticas prioritarias de desarrollo y docencia, así como la necesidad de inserción de todos los profesores del Departamento en alguna de estas líneas. Hacia el 2008 se observa que existían 18 proyectos patrocinados por el Departamento -en 2003 eran sólo 7-, 11 de los cuales contaban con financiamiento externo a la Universidad (nacional e internacional). Dichas iniciativas buscan entonces insertarse en las líneas de investigación definidas como prioritarias, a saber: estructura social y movimientos 
sociales; modernización del Estado y políticas públicas; ciudadanía, espacio público y espacio privado; metodología y técnicas de investigación social, y cambio cultural y relaciones sociales. Cabe constatar que desde el 2005 a la fecha han surgido nuevas áreas de trabajo, lo que contribuye a un continuo aumento del volumen de investigaciones y a una paulatina incorporación de estudiantes de pre y postgrado como asistentes y/o tesistas.

Los hallazgos en materia de preferencias temáticas arrojan que los macrotemas más trabajados corresponden a "política y poder", "género" y "economía" (tabla 2). Campos que experimentan un alza notable en el segundo período, y que se constituyen como temas en auge, son los de "cultura e identidad" y de "educación". Se observa el desarrollo de tópicos emergentes como "etnias", "derecho" y "medio ambiente", al tiempo que decrecen significativamente áreas como la sociología organizacional y la psicología social. Al dividir las apariciones como primera mención entre el total de apariciones de cada tema, obtenemos un coeficiente que permite identificar aquellos tópicos que resultan más centrales en los trabajos, por contraposición a aquellos cuya mención es periférica o tangencial. Así, por ejemplo, los temas económicos muestran un alto grado de centralidad, seguido de ámbitos urbanos, de cultura e identidad y de educación, todos los cuales podrían ser considerados campos de investigación consolidados junto a los estudios en estratificación social. A la inversa, temas como los medioambientales, edades o psicología social, tienen un carácter secundario y podrían considerarse variables de campos de estudio más que objetos de investigación por sí mismos.

Utilizando este mismo indicador es posible identificar las metodologías privilegiadas en cada campo. Se observa, en este sentido, una asociación entre el uso de estrategias cualitativas y la investigación en temas "urbanos", de "género", "salud", "rurales" y "cultura e identidad". Las estrategias cuantitativas, por su parte, tienden a identificarse en temas asociados a la "marginalidad", "economía", "educación", "política y poder" y estudios "organizacionales" (gráfico 3).

Cabe destacar que en el período de 2001 a 2007 existe una disminución relativa del peso de las estrategias cuantitativas entre los distintos macrotemas, excepto en los trabajos sobre marginalidad. Ahora bien, el predominio de estrategias de investigación cualitativas, por sobre estrategias mixtas, cuantitativas y/o teóricas, ha sido estable a lo largo de los 20 años entre los distintos ámbitos temáticos estudiados. Pese a ello, las asociaciones entre campos temáticos y diseños metodológicos no son significativas y reflejarían, una vez más, que los métodos son utilizados en base a criterios prácticos diseñados en el proceso mismo de construcción de la memoria de título.

\subsection{Referencias teóricas}

Como se señaló anteriormente, en la investigación también se consideró como ítem relevante las referencias bibliográficas; indicador que permite identificar a qué autores recurren con más frecuencia los tesistas a la hora de elaborar un marco teórico o abordar conceptualmente una problemática particular. El registro se realizó identificando los cinco 
TABLA 2

Memorias de titulación según macrotemas, por período (en porcentaje)

\begin{tabular}{|c|c|c|c|c|c|}
\hline \multirow{2}{*}{ MACROTEMAS } & \multicolumn{2}{|c|}{ PERÍODOS } & \multirow{2}{*}{ TOTAL*(A) } & \multirow{2}{*}{$\begin{array}{c}\text { TOTAL } 1^{\mathrm{a}} \\
\text { MENCIÓN**(B) }\end{array}$} & \multirow{2}{*}{$\begin{array}{c}\text { COEFICIENTE DE } \\
\text { CENTRALIDAD(B/A) }\end{array}$} \\
\hline & $1988-2000$ & 2001-2007 & & & \\
\hline Economía & 20,5 & 19,7 & 20,0 & 18,1 & 0,91 \\
\hline Política y poder & 22,9 & 25 & 24,2 & 14,4 & 0,58 \\
\hline Urbana & 4,8 & 6,1 & 5,6 & 3,7 & 0,66 \\
\hline Rural & 6 & 3 & 4,2 & 1,9 & 0,45 \\
\hline Cultura e identidad & 10,8 & 18,9 & 15,8 & 10,2 & 0,65 \\
\hline Marginalidad & 8,4 & 12,9 & 11,2 & 6 & 0,54 \\
\hline Metodología & 13,3 & 12,9 & 13,0 & 3,3 & 0,25 \\
\hline Género & 21,7 & 20,5 & 20,9 & 11,2 & 0,54 \\
\hline Edades & 9,6 & 7,6 & 8,4 & 1,4 & 0,17 \\
\hline Educación & 13,3 & 18,9 & 16,7 & 10,2 & 0,61 \\
\hline Organizacional & 14,5 & 7,6 & 10,2 & 2,8 & 0,27 \\
\hline Estratificación & 8,4 & 5,3 & 6,5 & 5,1 & 0,78 \\
\hline Etnias & 3,6 & 6,1 & 5,1 & 2,3 & 0,45 \\
\hline Derecho & 0 & 3 & 1,9 & 1,4 & 0,74 \\
\hline Salud & 6 & 5,3 & 5,6 & 4,2 & 0,75 \\
\hline Psicología Social & 13,3 & 3 & 7,0 & 1,4 & 0,20 \\
\hline Medio ambiente & 1,2 & 2,3 & 1,9 & 0 & 0,0 \\
\hline Teoría & 6 & 3,8 & 4,7 & 2,3 & 0,49 \\
\hline
\end{tabular}

* Las categorías no son mutuamente excluyentes, de modo que no suman 100\%.

** Las categorías corresponden al tema principal de la memoria, de modo que son excluyentes.

Fuente: elaboración propia. 


\section{GRÁFICO 3}

Memorias de titulación según macrotemas y estrategias metodológicas

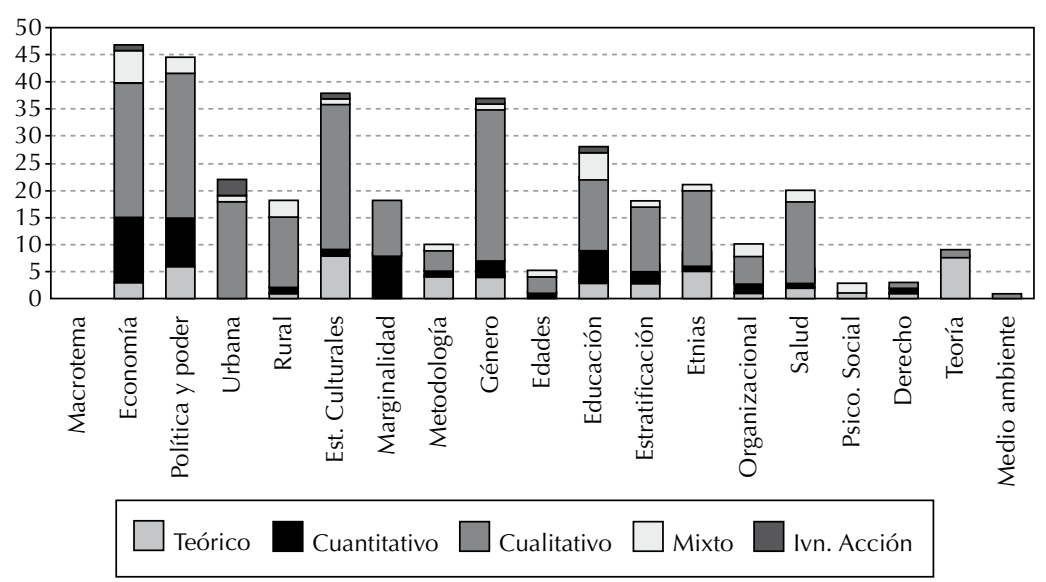

Fuente: elaboración propia.

autores más citados en los apartados teóricos de las memorias, de acuerdo al número total de referencias por autor. Del catastro se obtuvo una base de datos con 545 autores, lo cual da cuenta en primera instancia de una notable dispersión teórica y/o conceptual. Así lo demuestra el hecho de que parte importante de los autores mencionados tenga una sola aparición, aunque con el contrapunto del frecuente recurso a una veintena de autores.

Un caso paradigmático está representado sin duda por Pierre Bourdieu, quien se encuentra entre las primeras referencias en el 15,5\% del total de documentos, pasando de ser citado en el 5,8\% de éstas durante el período 1988-2000 a estarlo en el 26,1\% del siguiente período. Los datos muestran, además, que al realizar un cruce entre autor citado y macrotema del documento, se observa la transversalidad temática de las referencias al sociólogo galo. Otros autores ampliamente utilizados corresponden a Alain Touraine (9,1\%), Anthony Giddens (7,8\%), Max Weber (7,3\%) y Manuel Antonio Garretón (6,4\%), profesor del Departamento de Sociología y que es el único autor no europeo o norteamericano entre los primeros diez autores del listado (tabla 3).

A partir de los análisis realizados, es posible sostener dos tendencias gravitantes: los autores más citados son de origen preferentemente europeo y corresponden a aquellos que han realizado teorizaciones de carácter general o de amplio alcance; y la transversalidad temática de gran cantidad de ellos (son citados en memorias que abordan tópicos diversos entre sí), lo cual a su vez indica que los modelos conceptuales no son elaborados de manera rígida, sino que en la búsqueda de adecuaciones a problemáticas particulares y en muchos casos divergentes, lo cual da pie para hablar de la presencia de un cierto grado de eclecticismo teórico en las memorias de titulación. 
TABLA 3

Memorias de titulación según referencias bibliográficas, por período (en porcentaje)

\begin{tabular}{|c|c|c|c|}
\hline \multirow{2}{*}{ AUTOR } & \multicolumn{2}{|c|}{ PERÍODOS } & \multirow{2}{*}{ TOTAL* } \\
\hline & $1988-2000$ & 2001-2007 & \\
\hline Bourdieu, Pierre & 5,8 & 26,1 & 15,5 \\
\hline Touraine, Alain & 11,6 & 10,4 & 9,1 \\
\hline Giddens, Anthony & 7,2 & 10,4 & 7,8 \\
\hline Weber, Max & 14,5 & 5,2 & 7,3 \\
\hline Garretón, Manuel Antonio & 4,3 & 9,6 & 6,4 \\
\hline Berger, Peter & 10,1 & 5,2 & 5,9 \\
\hline Foucault, Michel & 8,7 & 6,1 & 5,9 \\
\hline Durkheim, Emile & 5,8 & 7,0 & 5,5 \\
\hline Habermas, Jürgen & 7,2 & 6,1 & 5,5 \\
\hline Merton, Robert & 11,6 & 3,5 & 5,5 \\
\hline Mead, George Herbert & 11,6 & 2,6 & 5,0 \\
\hline Larraín, Jorge & 2,9 & 7,0 & 4,6 \\
\hline Luhmann, Niklas & 5,8 & 5,2 & 4,6 \\
\hline Parsons, Talcott & 13,0 & 0,9 & 4,6 \\
\hline Castells, Manuel & 1,4 & 7,0 & 4,1 \\
\hline Ritzer, George & 5,8 & 3,5 & 3,7 \\
\hline Duarte, Claudio & 0,0 & 6,1 & 3,2 \\
\hline Marx, Karl & 4,3 & 3,5 & 3,2 \\
\hline
\end{tabular}

* Las categorías no son mutuamente excluyentes, de modo que no suman $100 \%$. Fuente: elaboración propia. 


\section{CONCLUSIONES}

En el marco de una agenda de investigación en torno a la reflexividad en las ciencias sociales, el artículo ha buscado instalar a las memorias de titulación como un producto indicativo, a la vez que particular, de la producción sociológica actual. Esto es así, al menos, para diseños organizacionales como los de la Universidad de Chile, donde la elaboración de estos documentos representa aún la única vía de acreditación del título profesional de sus egresados.

De la exposición de las tendencias en los trabajos de titulación durante los últimos veinte años, es plausible sugerir una discusión en relación a los condicionamientos que modelan las tesis en tanto producciones científicas orientadas a su comunicación en el sistema ciencia. En un principio, se sugirió que estas producciones se inscribían en una doble lógica. La primera, que podríamos abordar desde una perspectiva "internalista", obedece a desarrollos de la sociología y las ciencias sociales en general, los que están marcados por modelos paradigmáticos en el quehacer intelectual de la disciplina. En términos sistémicos, se trata de variaciones en los programas que seleccionan comunicaciones científicas en una u otra dimensión, y que, en tanto conforman la estructura del sistema, van variando sin que éste pierda su identidad como sistema clausurado en sus propias operaciones.

Claudio Ramos y su equipo de investigadores han puesto en evidencia la presencia de paradigmas que encajarían con la producción de investigaciones empíricas en sociología actual (Ramos, 2005); marco que ha sido extendido para la interpretación de los productos en antropología social y ciencia política (Ramos et al., 2008). Un abordaje como éste no ha sido consistente con los hallazgos arrojados en el análisis de memorias de título, lo que se debería, creemos, a la presencia de dos rasgos destacados. El primero de ellos dice relación con los criterios de adecuación metodológica de las investigaciones. La revisión de 220 textos mostró cómo los apartados metodológicos suelen elaborarse a través de una lógica práctica, altamente pragmática a los requerimientos del trabajo in situ. No hay clara asociación entre ellas y los campos temáticos o las preferencias teóricas del documento, y menos aún se hace explícita una opción epistemológica congruente.

Junto a lo anterior, los procedimientos de construcción teórica evidenciaron la fuerte dispersión de autores referenciados, aunque con una primacía de teóricos contemporáneos europeos (anglosajones, alemanes y franceses). Desde un punto de vista "externalista", esta importación teórica en parte es explicada por la presencia de figuras docentes en las unidades académicas, que instalan lógicas de trabajo que, sin alcanzar el estatuto de programas progresivos de investigación, sí al menos aumentan las probabilidades de aparición de ciertas corrientes teóricas. Ahora bien, la experiencia con el material escrito privilegia una interpretación en torno al eclecticismo teórico y al uso indistinto de autores de diversas escuelas de pensamiento. Aun en los casos en que pareciera haber una sólida construcción teórica, ésta viene dada a estudios de carácter metateórico y ensayísticos, y rara vez acompaña procesos de investigación empírica.

La cercanía que tienen las memorias de título con tendencias emergentes en los programas que la propia ciencia sociológica posee para la elección de ciertos temas, así como su contacto 
directo con las dinámicas del campo bajo el cual una comunidad concreta de practicantes ejerce su trabajo, hacen de estos documentos un indicador especialmente sensible a la apertura de nuevos ámbitos de estudio. Es así que exploraciones por esta vía pueden aportar información valiosa acerca de la vigencia de ciertos dominios de trabajo, la coexistencia de ámbitos de estudio disímiles y la aparición de noveles agendas de trabajo en la sociología del Chile actual.

\section{BIBLIOGRAFÍA}

Arnold, Marcelo (2004): "Introducción a las epistemologías sistémico constructivistas", en Francisco Osorio (ed.): Ensayos sobre socioautopoiesis y epistemología constructivista, Ediciones MAD, Santiago, pp. 7-15.

(2006): “Fundamentos de la observación de segundo orden", en Manuel Canales (coord.): Metodologías de investigación social. Introducción a los oficios, LOM Ediciones, Santiago, pp. 321-348.

(2009): "Comentarios sobre el estado de las ciencias sociales en Chile", Mesa Redonda: El estado de las ciencias sociales en Chile, Seminario sobre ciencias sociales y transdisciplinariedad, Facultad de Humanidades, Universidad de Valparaíso, sede Santiago, 5 de noviembre.

Arnold, Marcelo, Carlos Haefner, Daniel Quiroz y Mario Radrigán (1990): La antropología social en Chile. Producciones y representaciones, Universidad de Chile, Santiago.

Baño, Rodrigo (1984): “Nuevos estilos y nuevos temas en los análisis de ciencias sociales en la última década", Material de Discusión, №60, FLACSO, Santiago.

Barrios, Alicia y José Joaquín Brunner (1988): La sociología en Chile. Instituciones y practicantes, FLACSO, Santiago.

Bourdieu, Pierre (2000a): "El campo científico", en Intelectuales, política y poder, Eudeba, Buenos Aires, pp. 75-110.

(2000b): Los usos sociales de la ciencia, Nueva Visión, Buenos Aires.

(2000c): "La causa de la ciencia. Cómo la historia social de las ciencias sociales puede servir al progreso de estas ciencias", en Intelectuales, política y poder, Eudeba, Buenos Aires, pp. 111-127.

Brunner, José Joaquín (1985): "La participación de los centros académicos privados", Estudios Públicos, № 19 , pp. 1-12.

(1988): El caso de la sociología en Chile, FLACSO, Santiago.

(1989): Ciencias sociales y Estado: reflexiones en voz alta, FLACSO, Santiago.

Bunge, Mario (1993): Sociología de la ciencia, Siglo Veinte, Buenos Aires.

Comisión Académica Departamento de Sociología (2004): Diagnóstico y propuesta de reestructuración, Departamento de Sociología, Universidad de Chile, Santiago.

Courard, Hernán y Alicia Frohman (1999): Universidad y ciencias sociales en Chile, 1990-1995, Nueva Serie FLACSO, Santiago.

De Sierra, Gerónimo, Manuel Antonio Garretón, Miguel Murmis y Hélgio Trindade (2005): Las ciencias sociales en América Latina en una mirada comparativa, Proyecto Maison des Sciences de l'Homme, París. 
Departamento de Sociología (2009): Memoria anual de investigación 2008, Departamento de Sociología, Universidad de Chile.

Farías, Fernando (2004): "La sociología chilena en la década de los noventa", Cinta de Moebio, (19). (2009): "La epistemología de las ciencias sociales en la formación de competencias del pregrado", Cinta de Moebio (34), pp. 58-66.

Fernández, Enrique (2007): “Universidad y reconcentración de la investigación científica en Chile, 1982-2005", Persona y Sociedad, XXI (3), pp. 31-57.

Garretón, Manuel Antonio y Orlando Mella (eds.) (1995): Dimensiones actuales de la sociología, Bravo y Allende Editores, Santiago.

Garretón, Manuel Antonio (2005): "Social sciences and society in Chile: institutionalization, breakdown and rebirth", Social Science Information, 44 (2-3), pp. 359-409.

Luhmann, Niklas (1996): La ciencia de la sociedad, Universidad Iberoamericana, Iteso, Anthropos, México D.F.

Martínez, Andrés y Francy Ríos (2006): “Los conceptos de conocimiento, epistemología y paradigma, como base diferencial en la orientación metodológica del trabajo de grado", Cinta de Moebio (25).

Pinilla, Juan Pablo, Francisco Godoy, Tania Manríquez, Luis Vargas y Bernardita Ihnen (inédito): Memorias de titulación en antropología Social y sociología de la Universidad de Chile, 1988-2006, Documento de Trabajo, Línea reflexividad de las ciencias sociales, Programa Pulso-Departamento de Sociología, Universidad de Chile.

Ramos, Claudio (2005): "Cómo investigan los sociólogos chilenos en los albores del siglo XXI", Persona y Sociedad, XIX (3), pp. 85-119.

(2008): “¿Sistema, campo de lucha o red de traducción y asociaciones? Tres modelos para investigar la ciencia social y un intento de integración", Persona y Sociedad, XXII (2), pp. 9-52.

Ramos, Claudio, Andrea Canales y Stefano Palestini (2008): “El campo de las ciencias sociales en Chile: ¿Convergencia disciplinar en la construcción del objeto de estudio?", Cinta de Moebio (33), pp. 171-194.

Toledo-Nickels, Ulises (2008): “El esquema metateórico de Ritzer desde la metodología de los programas de investigación", Cinta de Moebio (33), pp. 204-218.

Wallerstein, Immanuel (1999): "El legado de la sociología, la promesa de la ciencia social”, en Roberto Briceño León y Heinz R. Sonntag (eds.): Legado de la sociología, la promesa de la ciencia social, Editorial Nueva Sociedad, Caracas, pp. 11-61.

(2004): "The three meanings of 'discipline'", en OECD: Re-inventing the social sciences, OECD publisher, París, pp. 22-25.

Weber, Max (1967): "La ciencia como vocación", en El político y el científico, Alianza Editorial, Madrid, pp. 180-231. 